\title{
HOMOTOPY GROUPS AND H-MAPS
}

\author{
by ALAIN JEANNERET \\ (Received 21st January 1994)
}

\begin{abstract}
The first nonvanishing homotopy group of a finite $\mathrm{H}$-space $X$ whose $\bmod 2$ homology ring is associative occurs in degrees 1,3 or 7 . Generators of these groups can be represented by maps $\alpha: S^{n} \rightarrow X$ for $n=1,3$ or 7 . In this note we prove that under some hypothesis on $X$ there exists an $H$-structure on $S^{n}, n=1,3$ or 7 such that $\alpha$ is an H-map.
\end{abstract}

1991 Mathematics subject classification: 55P45, 55Q05, 55Q52.

\section{Introduction}

Recently, J. P. Lin and the author [7] have proven that the first nonvanishing homotopy group of a finite $\mathrm{H}$-space whose $\bmod 2$ homology ring is associative occurs in degrees 1,3 or 7 . (Recall that a finite $\mathrm{H}$-space is an $\mathrm{H}$-space whose integral homology is finitely generated as a graded abelian group). This result improves Adams' famous theorem [1] saying that a sphere $S^{n}$ admits an H-structure if and only if $n=1,3$ or 7 .

The goal of this paper is to discuss the following question. If $(X ; \mu)$ is a finite $\mathrm{H}$-space, where $\mu$ is the multiplication on $X$, does there exist a set of generators of the first nonvanishing homotopy group $\left\{\alpha_{i}\right\} \subset \pi_{n} X, n=1,3$ or 7 and a multiplication $m$ on $S^{n}$ such that

$$
\alpha_{i}:\left(S^{n} ; m\right) \rightarrow(X ; \mu)
$$

are H-maps? (We will always identify a map with its homotopy class). The question can be easily answered in the case where $n=1$. The proofs are given in the next sections.

Theorem 1.1. If $X$ is a finite $H$-space, then there exists a set of generators $\left\{\alpha_{i}\right\}_{i=1, \ldots, n}$ of $\pi_{1} X$ such that

$$
\alpha_{i}: S^{1} \rightarrow X
$$

are $H$-maps for all $i=1, \ldots, n$.

We do need to specify the $H$-structure on $S^{1}$ because $S^{1}$ admits only one multiplication. 
The case where $n=3$ was first investigated by J. Schiffmann [13]; more precisely he has proven the following theorem.

Theorem 1.2. Let $X$ be a finite $H$-space carrying an homotopy associative multiplication such that $\pi_{3} X \cong \mathbf{Z}$. Let $\mu$ be a multiplication on $X$ and $\alpha \in \pi_{3} X$ a generator, then there exists a multiplication $m$ on $S^{3}$ such that

$$
\alpha:\left(S^{3} ; m\right) \rightarrow(X ; \mu)
$$

is an H-map.

Note that if $X$ is a finite $\mathrm{H}$-space, $\pi_{3} X$ is always a free abelian group. Even if the associative multiplication does not appear in the conclusion of the theorem, it is strongly used to prove it.

We can improve Schiffmann's result for a wider class of H-spaces, namely the nonassociative ones.

Theorem 1.3. Let $(X ; \mu)$ be a finite $H$-space with $\pi_{3} X \cong Z$ and $\alpha \in \pi_{3} X$ a generator, then there exists a multiplication $m$ on $S^{3}$ such that

$$
\alpha:\left(S^{3} ; m\right) \rightarrow(X ; \mu)
$$

is an H-map.

As an obvious corollary we can offer:

Corollary 1.1. Let $(X ; \mu),\left(X_{i} ; \mu_{i}\right), i=1, \ldots, n$ be finite $H$-spaces with $\pi_{3} X_{i} \cong \mathbf{Z}$ and $X \simeq \prod_{i=1}^{n} X_{i}$ as $H$-spaces. Then there exists a set of generators $\left\{\alpha_{i}\right\}_{i=1 \ldots \ldots n}$ of $\pi_{3} X \cong Z^{n}$ and multiplications $m_{i}, i=1, \ldots, n$ on $S^{3}$ such that

$$
\alpha_{i}:\left(S^{3} ; m_{i}\right) \rightarrow(X ; \mu)
$$

are H-maps.

Let us pause to comment:

(1) Many $\mathrm{H}$-spaces do not admit homotopy associative multiplication. For example if $G$ is a one-connected simple Lie group different from $G_{2}$ or $\operatorname{Spin}(7)$, then $G \times S^{7}$ does not carry a homotopy associative multiplication [4].

(2) All finite $\mathrm{H}$-spaces do not satisfy the splitting condition $X \simeq \prod X_{i}$ with $\left(X_{i} ; \mu_{i}\right)$ a finite $\mathrm{H}$-space, hence the corollary does not apply for all finite $\mathrm{H}$-spaces.

(3) Unlike $S^{1}$ which has a unique $H$-structure, $S^{3}$ has many [6], therefore the multiplications $m_{i}$ on $S^{3}$ depend on the generators $\alpha_{i}$.

(4) The situation for compact Lie groups is much nicer because problems of type occurring in (2) and (3) do not appear, cf. below. 
If $G$ is a simply connected compact Lie group then $G$ splits as $G \simeq \prod_{k=1}^{n} G_{k}$ with $G_{k}$ simple Lie groups. Let $H$ be any simple Lie group. It is well known that for any root $\rho$ of $H$ there exists a subgroup $S U(2)=S^{3}$ of $H$ [12]. The inclusion $i: S^{3} \rightarrow H$ is not necessarily a generator of $\pi_{3} H \cong Z$, but if the root $\rho$ is dominant [12], then $i$ is a generator of $\pi_{3} H$. If we come back to the simply connected Lie group $G$ with decomposition $G \cong \prod_{k=1}^{n} G_{k}$, it is obvious then there exists a set of generators $\left\{\alpha_{k}\right\}_{k=1, \ldots, n}$ of $\pi_{3} G \cong \mathbf{Z}^{n}$ such that

$$
\alpha_{k}: S U(2)=S^{3} \rightarrow G
$$

are group homomorphisms.

Before closing the introduction let us discuss the case of a 6-connected finite H-space. During many years the only known 6-connected finite $\mathrm{H}$-spaces were products of 7dimensional spheres. Recently Dwyer and Wilkerson have built up an "exotic" $\mathrm{H}$-space at the prime 2 [3]. More precisely they constructed a mod 2 finite $\mathrm{H}$-space $D I(4)$ whose $\bmod 2$ cohomology ring satisfies

$$
H^{*} D I(4) \cong \mathbf{F}_{2}\left[x_{7}\right] /\left(x_{7}^{4}\right) \otimes E\left(x_{11}, x_{13}\right)
$$

See [3] for the details. Using Zabrodsky's techniques of mixing homotopy types we can mix the 2-type of $D I(4)$ with $S^{7} \times S^{11} \times S^{27}$ localised at the set of all odd primes to construct an $\mathrm{H}$-space $X(4)$ whose mod 2 cohomology ring satisfies

$$
H^{*} X(4) \cong H^{*} D I(4)
$$

and which has no $p$-torsion in integral homology for odd primes $p$ (note that $\left.H^{*}(D I(4) ; \mathrm{Q}) \cong E\left(x_{7}, x_{11}, x_{27}\right)\right)$. In particular $\pi_{7} X(4) \cong \mathbf{Z}$. We can state now our last proposition.

Proposition 1.1. Let $\mu$ be a multiplication on $X(4)$ and $\alpha$ a generator of $\pi_{7} X(4) \cong Z$. Then there exists a multiplication $m$ on $S^{7}$ such that

$$
\alpha:\left(S^{7} ; m\right) \rightarrow(X(4) ; \mu)
$$

is an H-map.

\section{Proof of Theorem 1.1}

The fundamental group of $X$ is abelian and satisfies $\pi_{1} X \cong \bigoplus_{k=1}^{n} C_{k}$ with $C_{k}$ a cyclic group. We start with the situation where $\pi_{1} X$ is an infinite cyclic group, i.e. $\pi_{1} X \cong Z$. If $\pi_{1} X \cong Z$, it is well known that $X \cong S^{1} \times Y$ with $\pi_{1} Y=\pi_{2} Y=0$ cf. [11, page 63]. Therefore there exists a cellular decomposition of $X$ such that its 2-skeleton, $X^{(2)}$, is just $S^{1}$ [5]. Let $\alpha$ be a geneator of $\pi_{1} X$ that is identified with the inclusion $S^{1}=X^{(2)} \rightarrow X$. The composition 


$$
S^{1} \times S^{1 \stackrel{\alpha \times \alpha}{\longrightarrow}} X \times X \stackrel{\mu}{\longrightarrow} X
$$

factors through $X^{(2)}=S^{1}$. We have thus constructed a map $m: S^{1} \times S^{1} \rightarrow S^{1}$ such that the following diagram commutes

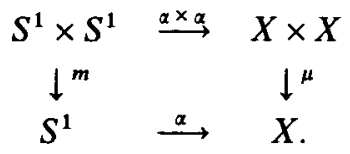

Hence $\alpha$ is an H-map.

The second situation is when $\pi_{1} X$ is a finite cyclic group i.e. $\pi_{1} X \cong Z / d$. In this case standard techniques show that $H_{1}(X ; Z) \cong Z / d, H^{1}(X ; \mathbf{Z})=0, H^{2}(X ; \mathbf{Z}) \cong \mathbf{Z} / d$. Let $x$ be a generator of $H^{2}(X ; Z)$. For dimensional reasons $x$ is a primitive class, and so the map $f: X \rightarrow K(Z ; 2)$ representing $x$ is an $H$-map. The fiber $F$ of $f$ is then an H-space.

If $\alpha$ is a generator of $\pi_{1} X$ then $\alpha: S^{1} \rightarrow X$ lifts to $\tilde{\alpha}: S^{1} \rightarrow F$. It is sufficient to prove that $\tilde{\alpha}$ is an $\mathrm{H}$-map because the inclusion $i: F \rightarrow X$ is an H-map and by construction $\alpha=i \circ \tilde{\alpha}$. Now observe that $F$ is a finite $\mathrm{H}$-space with $\pi_{1} F \cong \mathbf{Z}$ generated by $\tilde{\alpha}$. Using the first case discussed above we obtain that $\tilde{\alpha}$ is an H-map.

Let us consider now the general case $\pi_{1} X \cong \bigoplus_{k=1}^{n} C_{k}, C_{k}$ a cyclic group. Let $p_{k}: X_{k} \rightarrow X$ be the covering space such that $\pi_{1} X_{k} \cong C_{k}$. The covering map $p_{k}$ is an H-map. Then there exists a generator $\tilde{\alpha}_{k}$ of $\pi_{1} X_{k}$ which is an H-map. The set $\left\{\alpha_{k}\right\}_{k=1, \ldots, n}$ with $\alpha_{k}=p_{k} \circ \tilde{\alpha}_{k}$ is a set of generators of $\pi_{1} X$ and all the $\alpha_{k}$ are H-maps.

\section{Proof of Theorem 1.3}

First remark that $X$ is not assumed to be 1 -connected. The universal cover $\tilde{X}$ of $X$ satisfies $\pi_{3} \tilde{X} \cong \pi_{3} X$ and $\tilde{X}$ has the homotopy type of a finite $H$-space. We can therefore assume through all the proof that $X$ is 2 -connected and $\pi_{3} X \cong Z$.

A result of Lin [9] asserts that

$$
Q H^{\text {even }}\left(X ; \mathbf{F}_{p}\right) \cong \sum_{i=1}^{\infty} \beta_{1} \mathscr{P}^{i} H^{2 n_{i}+1}\left(X ; \mathbf{F}_{p}\right)
$$

where $p$ is an odd prime, $Q H^{\text {even }}$ is the module of indecomposables in even dimensions, $\beta_{1}$ is the first Bockstein and $\mathscr{P}^{i}$ is the $i^{\text {th }}$ Steenrod power. In our situation this result implies that $H^{k}(X ; Z)$ has no $p$-torsion for $p$ an odd prime and $k \leqq 6$. Hence we are reduced to study the 2-torsion of the integral cohomology in low dimensions.

To simplify the notations, $H^{*} X$ will stand for $H^{*}\left(X ; \mathbf{F}_{2}\right)$. Recall two results due to Kane and Lin.

Theorem 3.1 [8]. Let $X$ be a simply connected mod 2 finite $H$-space, then

$$
Q H^{\text {even }} X=0 \text {. }
$$


Theorem 3.2 [10]. Let $X$ be a simply connected mod 2 finite $H$-space, then

$$
Q H^{4 k+1} X=\mathrm{Sq}^{2 k} Q H^{2 k+1} X, k \geqq 1
$$

We apply these results in our particular case. The first result implies that $H^{4} X=$ $Q H^{4} X=0$ because $H^{2} X=0, X$ being 2-connected. Moreover $H^{6} X \cong \xi H^{3} X$ with $\xi$ the cup square map. The second result implies that $H^{5} X \cong \mathrm{Sq}^{2} H^{3} X$. Hence $\xi H^{3} X \cong$ $\mathrm{Sq}^{3} H^{3} X \cong \mathrm{Sq}^{1} \mathrm{Sq}^{2} H^{3} X \cong \mathrm{Sq}^{1} H^{5} X$. So three different cases can occur:

1. $H^{6} X \neq 0$ and so $H^{6} X \cong H^{5} X \cong H^{3} X \cong \mathrm{F}_{2}$, if we set $x_{k}$ a generator of $H^{k} X$ then

$$
\mathrm{Sq}^{2} x_{3}=x_{5}, \mathrm{Sq}^{1} x_{5}=x_{6}=x_{3}^{2} \text {. }
$$

2. $H^{6} X=0, H^{5} X \neq 0$ and so

$$
\mathrm{Sq}^{2} x_{3}=x_{5}, \mathrm{Sq}^{1} x_{5}=0
$$

3. $H^{5} X=0$ and so

$$
\mathrm{Sq}^{2} x_{3}=0
$$

We will prove the theorem under the conditions stated in (1), the cases (2) and (3) being similar or simpler.

Let $K_{0}=K\left(\mathrm{~F}_{2} ; 5\right)$ and $h_{0}: X \rightarrow K_{0}$ the classifying map of $x_{5}$ the generator of $H^{5} X$. Define $E_{0}$ to be the fiber of $h_{0}$. The Serre spectral sequence applied to the fibration

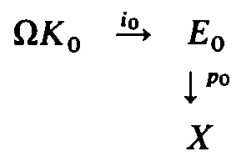

implies that

$$
H^{j} E_{0} \cong \begin{cases}\mathbf{F}_{2} & \text { if } j=3,6 \\ 0 & \text { if } j=1,2,4,5\end{cases}
$$

and $i_{0}^{*}: H^{6} E_{0} \cong H^{6} \Omega K_{0}$. Since the class $x_{5}$ is primitive, the map $h_{0}$ is an H-map, hence $E_{0}$ is an H-space and $i_{0}$ and $p_{0}$ are H-maps too. We call $a$ the generator of $H^{6} E_{0} \cong \mathrm{F}_{2}$. It satisfies $i_{0}^{*} a=\mathrm{Sq}^{2} \imath_{4}$, where $l_{4}$ is the fundamental class of $\Omega K_{0}=K\left(\mathrm{~F}_{2} ; 4\right)$. We will prove in the next section the central lemma.

Lemma 3.1. The class $a \in H^{6} E_{0}$ is primitive.

Now let $K_{1}=K\left(\mathrm{~F}_{2} ; 6\right)$ and $h_{1}: E_{0} \rightarrow K_{1}$ be the classifying map for $a$. Let $E_{1}$ be the fiber of $h_{1}$. The Serre spectral sequence of the fibration 


$$
\begin{array}{ll}
\Omega K_{1} \stackrel{i_{1}}{\rightarrow} & E_{1} \\
& \downarrow^{p_{1}} \\
& E_{0}
\end{array}
$$

implies that

$$
H^{j} E_{1} \cong \begin{cases}\mathbf{F}_{2} & \text { if } j=3 \\ 0 & \text { if } j=1,2,4,5,6\end{cases}
$$

In particular $E_{1}$ admits a cellular decomposition such that its 6-skeleton, $E_{1}^{(6)}$, is just $S^{3}$ [5]. The map $h_{1}$ is an H-map because the cohomology class $a$ is primitive, therefore $E_{1}$ is an $\mathrm{H}$-space; $i_{1}$ and $p_{1}$ are $\mathrm{H}$-maps.

Let $\alpha$ be a generator of $\pi_{3} X$, it is clear that $\alpha$ lifts in the following way:

$$
\begin{array}{ccccc} 
& \tilde{\alpha} & E_{1} & & \\
& \nearrow & \downarrow_{p_{1}} & & \\
S^{3} & \stackrel{\hat{x}}{\rightarrow} & E_{0} & \stackrel{h_{1}}{\rightarrow} & K_{1} \\
& \searrow & \downarrow_{p_{0}} & & \\
& \propto & X & \stackrel{h_{0}}{\rightarrow} & K_{0} .
\end{array}
$$

The theorem will be proven as soon as we can exhibit a multiplication $m$ on $S^{3}$ such that $\tilde{\alpha}$ is an H-map, because $\alpha=p_{0} \circ p_{1} \circ \tilde{\alpha}$ and $p_{0}, p_{1}$ are H-maps. The argument is the following:

As claimed above $E_{1}$ admits a cellular decomposition such that $E_{1}^{(6)}=S^{3}$. We identify $\tilde{\alpha}$ with the inclusion $S^{3}=E_{1}^{(6)} \rightarrow E_{1}$. Let $\tilde{\mu}$ be a cellular multiplication on $E_{1}$ induced from the one on $X$. The composition

$$
S^{3} \times S^{3} \stackrel{\tilde{a} \times \tilde{a}}{\longrightarrow} E_{1} \times E_{1} \stackrel{\tilde{\mu}}{\longrightarrow} E_{1}
$$

factors through $E_{1}^{(6)}=S^{3}$. Therefore we have constructed a map $m: S^{3} \times S^{3} \rightarrow S^{3}$ such that the following diagram is commutative

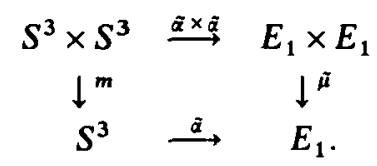

The restriction of $m$ to $S^{3} \vee S^{3}$ is homotopic to the folding map because the restriction of $\tilde{\mu}$ to $E_{1} \vee E_{1}$ is homotopic to the folding map as $\tilde{\mu}$ is a multiplication on $E_{1}$. So $m$ is a multiplication on $S^{3}$ and $\tilde{\alpha}$ is an $\mathrm{H}$-map. The theorem is proven.

\section{Proof of Lemma 3.1}

The fibration defined in the previous section 


$$
\begin{array}{llll}
E_{0} & & \\
\downarrow^{p 0} & & \\
X & \stackrel{n_{0}}{\rightarrow} & K_{0}
\end{array}
$$

induces an exact sequence in cohomology with $\mathbf{F}_{2}$ coefficients up to dimension 7. Recall that if $Y$ is an $H$-space then the projective plane of $Y$ [2] denoted by $P_{2} Y$ fits into a cofibration

$$
\Sigma Y \stackrel{i_{Y}}{\rightarrow} P_{2} Y \stackrel{k_{Y}}{\rightarrow} \Sigma Y \wedge \Sigma Y .
$$

The naturality of the cofibration allows us to construct the following commutative diagram:

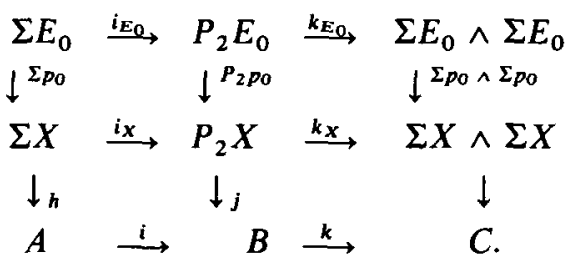

The spaces $A, B$ and $C$ are the cofibres of the maps $\Sigma p_{0}, P_{2} p_{0}, \Sigma p_{0} \wedge \Sigma p_{0}$ respectively. The sequence

$$
A \stackrel{i}{\rightarrow} B \stackrel{k}{\rightarrow} C
$$

is again a cofibration.

As $\left(\Sigma p_{0}\right)^{*}: H^{n} \Sigma E_{0} \cong H^{n} \Sigma X$ for $n \leqq 5$, we deduce that $H^{n} C=0$ for $n \leqq 10$. In particular $H^{n} A \cong H^{n} B$ for $n \leqq 9$. The remark made after $\left({ }^{* *}\right)$ allows us to identify

$$
H^{n} A \cong H^{n} B \cong H^{n} \Sigma K_{0}
$$

for $n \leqq 8$.

Let $x_{3} \in H^{3} X$ be the generator and $y_{3} \in H^{3} E_{0}$ be the class such that $p_{0}^{*} x_{3}=y_{3}$. For dimensional reasons $x_{3}$ and $y_{3}$ are primitive, so there exist $u_{4} \in H^{4} P_{2} X$ and $v_{4} \in H^{4} P_{2} E_{0}$ satisfying $i_{X}^{*} u_{4}=\sigma x_{3}, i_{E_{0}}^{*} v_{4}=\sigma y_{3}, \sigma$ standing as usual for the suspension isomorphism.

The top cofibration of $\left(^{* *}\right)$ can be written as

$$
P_{2} E_{0} \stackrel{k E_{0}}{\longrightarrow} \Sigma E_{0} \wedge \Sigma E_{0} \stackrel{\Sigma^{2} \mu}{\longrightarrow} \Sigma^{2} E_{0}
$$

with $\mu$ the multiplication on $E_{0}$. Recall from [2] that $\Sigma^{2} \mu$ induces the reduced coproduct in cohomology. The only possible non-trivial reduced coproduct for $a \in H^{6} E_{0}$ is $y_{3} \otimes y_{3}$ so $\left(\Sigma^{2} \mu\right)^{*} \sigma^{2} a=\sigma y_{3} \otimes \sigma y_{3}$ or $\left(\Sigma^{2} \mu\right)^{*} \sigma^{2} a=0$.

Browder and Thomas [2] have studied the cohomology ring structure of the projective plane. One of their results is that, in our situation, $\left(k_{E_{0}}\right)^{*} \sigma y_{3} \otimes \sigma y_{3}=v_{4}^{2}$. In 
particular we deduce from the exactness in cohomology of $(* * *)$ that $a$ is primitive if and only if $v_{4}^{2} \neq 0$ in $H^{8} P_{2} E_{0}$.

As $X$ is a finite $H$-space we already know [2] that $u_{4}^{2} \neq 0$ in $H^{8} P_{2} X$. The problem is that $E_{0}$ is not a finite $\mathrm{H}$-space and so $v_{4}^{2}$ is not automatically non-trivial.

Using the exact sequence in cohomology induced from

$$
P_{2} E_{0} \stackrel{p_{2} p_{0}}{\longrightarrow} P_{2} X \stackrel{j}{\longrightarrow} C
$$

we therefore just need to prove that $u_{4}^{2}$ is not in the image of $j^{*}$ (recall that $\left.\left(P_{2} p_{0}\right) * u_{4}=v_{4}\right)$.

Under the identification $H^{n} A \cong H^{n} \Sigma K_{0}, n \leqq 8$ the homomorphism $h^{*}$ coincides with $\left(\Sigma h_{0}\right)^{*}$ and $j^{*}$ with $\left(P_{2} h_{0}\right)^{*}$ up to dimension 8 .

By definition $h_{0}^{*} l_{5}=\mathrm{Sq}^{2} x_{3}=x_{5}$. So from commutativity of the diagram $\left({ }^{* *}\right)$ we get $j^{*} \sigma l_{5}=\left(P_{2} h_{0}\right)^{*} \sigma l_{5}=\mathrm{Sq}^{2} u_{4}$, hence $j^{*} \sigma \mathrm{Sq}^{2} l_{5}=\mathrm{Sq}^{2} \mathrm{Sq}^{2} u_{4}=0$. As $\sigma \mathrm{Sq}^{2} l_{5}$ is the only non-trivial element of $H^{8} \Sigma K_{0}$, we conclude that $\operatorname{Im}\left(P_{2} h_{0}\right)^{*}=0$ in dimension 8 , which finishes the proof of the lemma.

\section{Proof of Proposition 1.1}

The proof of Proposition 1.1 is completely analogous to the one of Theorem 1.3. Let us just mention the 2 stage Postnikov tower needed:

$$
\begin{array}{rllll} 
& \tilde{a} & E_{1} & & \\
& > & \downarrow_{p_{1}} & & \\
S^{7} & \stackrel{\tilde{\alpha}}{\rightarrow} & E_{0} & \stackrel{h_{1}}{\longrightarrow} & K\left(\mathrm{~F}_{2} ; 14\right) \\
& > & \downarrow_{p 0} & & \\
\tilde{\alpha} & X & \stackrel{h_{0}}{\rightarrow} & K(\mathrm{Z} ; 11) .
\end{array}
$$

The map $h_{0}$ is the classifying map for the class $x_{11}, h_{1}$ classifies the class $a \in H^{14} E_{0}$ whose restriction to the fiber of $p_{0}, \Omega K(\mathrm{Z} ; 11)=K(\mathrm{Z} ; 10)$ is $\mathrm{Sq}^{4} \imath_{10}$. Again $E_{0}$ and $E_{1}$ are H-spaces, moreover the 14-skeleton of $E_{1} E_{1}^{(14)}$ satisfies $E_{1}^{(14)}=S^{7}$. As before we construct the following commutative diagram

$$
\begin{array}{ccc}
S^{7}=S^{(7)} & \stackrel{\tilde{\alpha} \times \tilde{\alpha}}{\longrightarrow} E_{1} \times E_{1} \\
\downarrow^{m} & & \downarrow^{\tilde{\mu}} \\
S^{7}=E_{1}^{(14)} \longrightarrow & E_{1} .
\end{array}
$$

In this way $S^{7}$ is endowed with a multiplication $m$ such that $\tilde{\alpha}$ is an H-map, so $\alpha$ itself is an H-map.

\section{REFERENCES}

1. J. F. Adams, Vector fields on spheres, Ann. of Math. 75 (1962), 603-632. 
2. W. Browder and E. Thomas, On the projective plane of an H-space, Illinois J. Math. 7 (1963), 492-502.

3. W. G. Dwyer and C. W. Wilkerson, A new finite loop space at the prime two, J. Amer. Math. Soc. 6 (1993), 37-64.

4. D. L. Goncalves, Mod 2 homotopy associative H-spaces (Lecture Notes in Mathematics, 657, 1978), 198-216.

5. P. Hilton, Homotopy theory and duality (Gordon and Breach, 1965).

6. I. M. James, Multiplications on spheres III, Trans. Amer. Math. Soc. 84 (1957), 545-558.

7. A. Jeanneret et J. P. Lin, Connexité des H-spaces finis, C. R. Acad. Sci. Paris 315 (1992), 829-831.

8. R. M. Kane, Implications in Morava K-theory, Mem. Amer. Math. Soc. 340 (1986).

9. J. P. Lin, Torsion in H-spaces II, Ann. of Math. 107 (1978), 41-88.

10. J. P. LIN, $4 k+1$ dimensional generators of finite $\mathrm{H}$-spaces, manuscript.

11. N. Mahamed, R. Piccinini and U. Suter, Some Applications of Topological K-Theory (North-Holland Mathematics Studies 45, 1980).

12. M. Mimura and H. TOda, Topology of Lie groups I and II (Amer. Math. Soc. Translations of Mathematical Monographs, 1991).

13. S. J. Schiffman, A Samelson product and homotopy associativity, Proc. Amer. Math. Soc. 70 (1978), 189-195.

UNIVERSITÄT BERN

Mathematisches Institut

Sidlerstrasse 5

Ch-3012 BERN 